\title{
Expression of the Sweet Receptor Protein, T1R3, in the Human Liver and Pancreas
}

\author{
Kazumi TANIGUCHI ${ }^{1,2)}$ \\ ${ }^{1)}$ Department of Veterinary Anatomy, School of Veterinary Medicine and Animal Sciences, Kitasato University, 23-35-1 Higashi, Towada, \\ Aomori 034-8628, Japan and ${ }^{2)}$ Monell Chemical Senses Center, 3500 Market Street, Philadelphia, Pennsylvania 19104, U.S.A.
}

(Received 26 January 2004/Accepted 12 May 2004)

ABSTRACT. The expression of T1R3, a taste receptor essential for the perception of sweetness and umami-taste, was examined by immunohistochemistry to determine whether and where it may be localized in the liver and pancreas. In the liver, both immunopositive and immunonegative reactions were detected; bile ducts and intercalated portions of the bile ductules were immunopositive to T1R3, while arterioles and venules were immunonegative in interlobular connective tissue. In the hepatic lobule, all other cells including liver cells (hepatocytes) and bile capillaries were immunonegative. In the pancreas, all endocrine portions of the pancreas were immunonegative to T1R3. Within the exocrine portions, immunopositive reactions were detected in excretory duct cells, intercalated cells, and centroacinar cells. In contrast, acinar cells were immunonegative, as were vessels, lymph capillaries, nerve fibers, and connective tissue cells in the exocrine portions. The restricted localization of T1R3 in the duct cells of the liver and pancreas in the present study may indicate that T1R3 is involved in monitoring changes in the makeup of bile and pancreatic juices in the hepatic and pancreatic duct systems. KEY WORDS: liver, pancreas, sweet, taste, T1R3.

J. Vet. Med. Sci. 66(11): 1311-1314, 2004

Taste receptors have been classified into several groups: T1R family which senses sweetness and umami-taste, the T2R family involved in detecting bitter compounds, and ion channels which are responsible for detecting acidic and salty tastes $[3,5,9,10]$. T1R3 is a seven-transmembrane, $G$ protein-coupled receptor (GPCR) that has been identified as a component of a taste receptor essential for the perception of several types of sweeteners $[2,8,12,13,17]$. T1R3 was discovered as the third member of the T1R family. T1R3 forms a heterodimer with T1R1 or T1R2, other currently identified members of T1R family. The heterodimer of T1R1 and T1R3 functions for umami taste (amino acids and ribonucleotides) receptor $[8,14]$, and the heterodimer of T1R2 and T1R3 recognizes many sweet-tasting molecules such as sucrose and saccharin [8, 15]. Recently Ariyasu et al. (2003) suggested that T1R3 alone, perhaps as a monodimer, functions as a molecule essential for the perception of disaccharide trehalose [1]. In addition to taste cells on the tongue, T1R3 is also reported to be expressed in a wide variety of tissues such as brain, testis, liver, and lung by several Northern blot analysis [12] and RT-PCR studies [7]; although, the functional significance of T1R3 in these tissues is still unknown. Since T1R3 is related to task which in turn influences food intake, it may be involved in recognitional digestion of foods and be expressed in several digestive glands such as the liver. In this study, therefore, I examined the types of cells in which T1R3 is expressed in liver and pancreatic tissues, as representatives of digestive glands, and whether the T1R3 messenger RNA expressed in these organs is translated into protein-when the cell types are clarified, sometimes we can get better idea of the protein's roles.

\section{MATERIALS AND METHODS}

Sections: Paraffin sections of a human liver and pancreas were commercially obtained from a company which sold them with agreements of providers (Novagen: Human digestive system set, Cat. No. 70318, in Human Normal Hybrid-Ready Tissues, San Diego, U.S.A.). According to the manufacturer, these organs were removed, immersed immediately into a fixative-fresh phosphate-buffered saline (PBS) containing 4\% paraformaldehyde-, embedded in paraffin by routine procedures, and sectioned at 5 $\mu \mathrm{m}$.

In the present study, these sections were deparaffinized and processed for the immunohistochemical examinations.

Antibody against T1R3: An antibody was raised against T1R3 which was previously demonstrated in the taste buds [16]. I selected a sequence of amino acids, (N-end) STEEATLNQRAQPNSTLC (C-end), which was unique for T1R3 and exhibited no cross reaction to any known molecules including T1R1 and T1R2. Zymed Laboratories Inc (South San Francisco, U.S.A.) synthesized the amino acids accordingly, conjugated it with $\mathrm{KLH}$, and injected it into rabbits to raise the antibody. The production of anti-T1R3 antibody was confirmed by ELIZA and purified by affinity chromatography. As control experiments, the following two types of serum were utilized: (1) the serum of the same rabbits obtained before the injection of the antigen, and (2) the antiserum preliminarily incubated with excess amounts of the synthesized amino acids which were used as the antigen. No specific reaction was observed in these control experiments.

Immunohistochemistry procedures: The sections were deparaffinized by routine procedures and treated with $0.3 \%$ $\mathrm{H}_{2} \mathrm{O}_{2}$ in methanol for $30 \mathrm{~min}$ to eliminate endogenous peroxidase activity. They were incubated in $2 \%$ normal goat 
serum for $30 \mathrm{~min}$ at room temperature (RT) to block nonspecific binding, followed by anti-T1R3 antibody $(1: 1,000)$ at $4^{\circ} \mathrm{C}$ for 3 days. Then, they were treated with a secondary antibody, biotinylated goat anti-rabbit IgG (Histofine SABRO kit, Nichirei, Tokyo). The sections were then incubated with peroxidase-conjugated streptavidin-biotin complex (Nichirei) for $60 \mathrm{~min}$ at RT. After each step, the sections were rinsed thoroughly in $0.01 \mathrm{M}$ phosphate buffered saline ( $\mathrm{pH} 7.2$ ) containing $0.05 \%$ Tween 20 (Sigma). Immunoreaction products were obtained through reaction with $0.0125 \%$ diaminobenzidine (DAB) and $0.002 \% \mathrm{H}_{2} \mathrm{O}_{2}$ in $0.05 \mathrm{M}$ Tris-HCl buffer ( $\mathrm{pH}$ 7.6). The sections were counterstained with hematoxylin and viewed with a light micro-

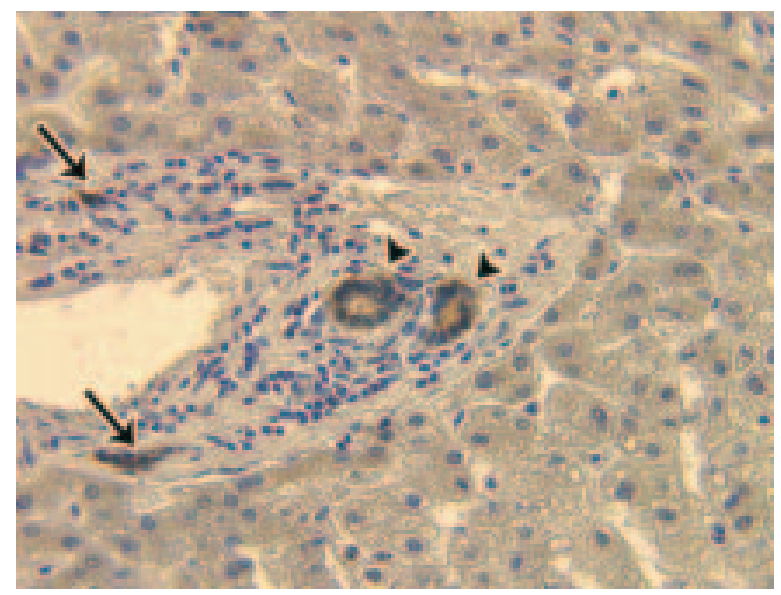

Fig. 1. Immunohistochemistry for T1R3 in the liver. Positive immunoreactions to T1R3 are observed in the interlobular secretory duct and intercalated ductules (Hering's duct) (arrow) in the interlobular connective tissue. The reaction products are ubiquitously distributed in the cytoplasm of the duct cells. Cellular nuclei are counter stained with hematoxylin. $\times 760$.

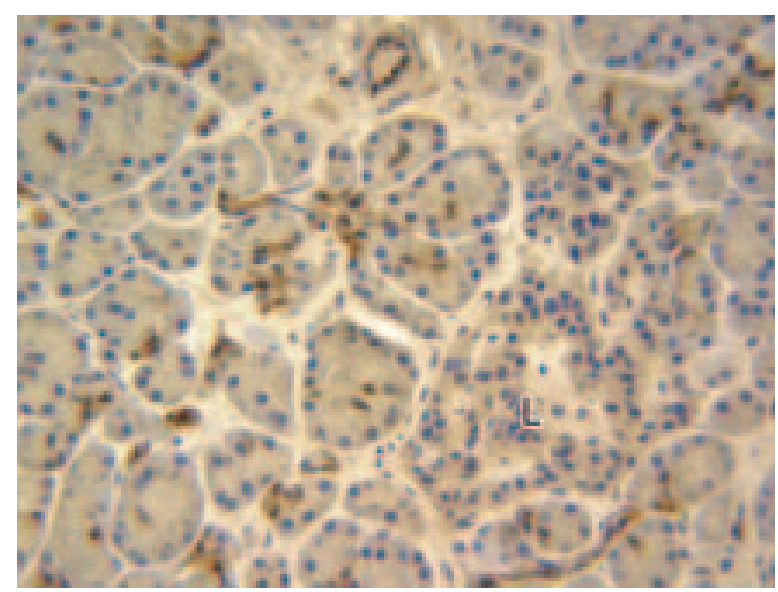

Fig. 2. Immunohistochemistry for T1R3 in the pancreas. Positive immunoreactions to T1R3 are observed in the exocrine portion of the pancreas and restricted to duct structures and the center of the acini, while pancreatic islets of Langerhans (L) are negative to T1R3. $\times 760$. scope (Nikon, Tokyo, Japan).

\section{RESULTS}

Liver: All of the cells constituting interlobular bile ducts located in the interlobular connective tissue (the Glisson's sheaths) were immunopositive to T1R3 (Fig. 1, arrowhead). In addition, intercalated portions of the bile ductules (Hering's duct) connecting bile capillaries with intercalated bile ducts were immunopositive in the interlobular connective tissue (Fig. 1, arrow). The immunopositive deposits were diffusely distributed in the cytoplasm of these duct cells. However, the other components of the hepatic triad in the interlobular connective tissues-the arterioles and venuleswere immunonegative to T1R3. Fibroblasts making up the major part of the connective tissue were also immunonegative to T1R3. None of the liver cells (hepatocytes), stellate cells (Kupffer's cells), Itoh lipid storing cells, nor endothelial cells, in the hepatic lobules were immunopositive to T1R3. The bile capillaries between hepatocytes were also not immunopositive.

Pancreas: All endocrine portions of the pancreas (pancreatic islets of Langerhans) were immunonegative to T1R3 (Fig. 2, L). Positive reactions, however, were detected in cells constituting excretory ducts in the exocrine pancreas (Fig. 2); tall intra-lobular duct cells and short inter-lobular duct cells, as well as intercalated cells, were immunopositive. Centroacinar cells were also immunopositive. Immunopositive deposits were ubiquitously distributed in the cytoplasm of all these cell types (Fig. 3, arrow). In contrast, acinar cells, which constitute the major part of the exocrine portions of the pancreas, were immunonegative, as were vessels, lymph capillaries, nerve fibers, and connective tissue cells in the exocrine portions.

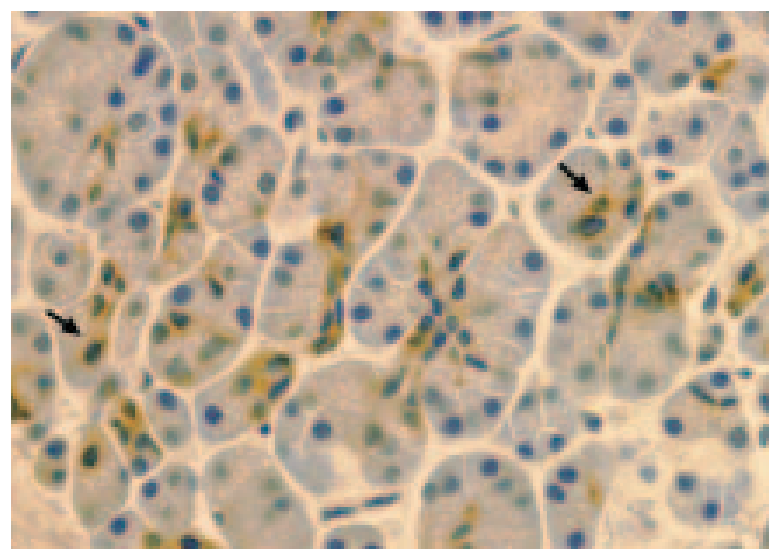

Fig. 3. Higher magnification of the immunohistochemistry for T1R3 in the pancreas. Intercalated duct cells as well as centroacinar cells (arrows) are immunopositive to T1R3, while acinar cells are negative to T1R3. $\times 760$. 


\section{DISCUSSION}

The present study demonstrates that: (1) the sweet receptor protein, T1R3, is expressed in the liver and pancreas cells, not only in the taste buds, and, (2) T1R3 is localized in duct cells in these digestive glands. Both the liver and pancreas play indispensable roles in the digestive and metabolic processes of the body. Since T1R3 was first identified as a receptor protein which senses sweetness in the food, it was most reasonable to hypothesize that this protein takes part in chemical sensation in duct cells in the liver and pancreas.

The present study has found a relationship between T1R3 and bile. In the liver, one of the important roles of liver cells (hepatocytes) is the secretion of bile. The bile secreted by hepatocytes flows into the bile capillary between the hepatocytes. The bile capillary is connected to the bile ductule, and the bile ductule to the interlobular bile duct. The present study reveals that the positive immunoreactions to T1R3 were restricted to the bile ductule cells and interlobular bile duct cells. These cells are directly exposed to the bile and function to detect substances therein. If ligands in the bile bind to T1R3 on the cellular membrane of the bile ductule and bile duct to provide information about the molecular components of the bile produced by hepatocytes, T1R3 may function to monitor changes in the components of the bile in the hepatic duct system. However, the hepatocytes and bile capillaries were immunonegative to T1R3. These results suggest that T1R3 is not synthesized in the hepatocytes, and that T1R3 functions as a receptor to detect substances in the bile in the interlobular space but not inside of the hepatic lobules.

In the pancreas, immunopositive reactions to $\mathrm{T} 1 \mathrm{R} 3$ are confined to both excretory duct cells and centroacinar cells, the latter considered to be a modified type of duct cell. These cells are exposed directly to the pancreatic juice in a similar manner as the bile ductule and bile duct cells which are exposed directly to the bile in the liver. Pancreatic acinar cells secreting the pancreatic juice were negative to T1R3 as were hepatocytes in the liver. These findings suggest that T1R3 is not synthesized in the cells which bear major functions in these digestive organs. In addition, as pancreatic islet cells were also negative to T1R3, it is quite difficult to postulate the participation of T1R3 in the endocrine function of the pancreas which includes the secretion of pancreatic hormones such as insulin or glucagon that control the blood glucose level. This suggests that T1R3 plays an additional role in the exocrine function of digestive glands other than sweetness perception. The present findings suggest that T1R3 in the pancreatic duct cells might be involved in chemosensory functions to detect ligands in the pancreatic juice similar to its role in hepatic duct cells. It may be further suspected that there are unknown molecules in both the bile and pancreatic juices which bind to T1R3.

There have been no previous reports of the expression of T1R3 in the pancreas. However, it has been reported that the pancreas contains a molecule related to the perception of bitterness, alpha-gustducin, which is also found in the tongue $[6,11]$. In this context, it can be said that at least two kinds of molecules related to the perception of taste are present in both the tongue and pancreas. Many problems remain to be resolved to reveal the physiological significance of the co-existence of these molecules in these organs.

To date, three members of the T1R family, T1R1, T1R2 and T1R3, have been identified and studied. The heterodimer of T1R1 and T1R3 functions as a umami-taste (amino acids) receptor and that of T1R2 and T1R3 as a sweetness receptor, including sucrose, saccharin, dulcin, acesulfame-K, brazzein, monellin and thaumatin $[8,14,15$, 18]. In addition, Demak et al. (2003) suggested that T1R3independent sweet- and umami-responsive receptors exist on the tongue [4]. Moreover, recently Ariyasu et al. (2003) demonstrated that T1R3 was an essential molecule in recognizing the disaccharide trehalose [1]. They revealed that both of the cell types expressing T1R1+T1R3 and T1R2+T1R3 responded to trehalose; moreover, T1R3 alone also responded to the trehalose. Their results suggested that T1R3 does not necessarily require T1R1 or T1R2 to reveal its function; it may function as a single molecule or homodimer. In my preliminary studies using antibodies against T1R1 and T1R2, I did not find any positive immunoreaction to T1R1 or T1R2 in the liver and pancreas (data not shown). The positive immunoreaction of T1R3 in the present study suggests that T1R3 may be present in the liver and pancreas as a form of homodimer or a heterodimer with an unknown molecule but not with T1R1 or T1R2, and that it plays an as yet undefined role, perhaps, to monitor substances in the secretory products of these organs.

In conclusion, the sweet receptor T1R3 is expressed in duct cells in the liver and pancreas. T1R3 was first identified as a receptor protein which senses sweetness in food. I suggest that this protein also takes part in chemical perception in duct cells in the liver and pancreas, perphaps as a homodimer, to regulate digestion by playing a role in chemosensory processes related to bile and pancreatic juices.

ACKNOWLEDGEMENTS. I express my sincere gratitude to Dr. Gary K. Beauchamp, the director of Monell Chemical Senses Center, Philadelphia, U.S.A., for his critical reading of this manuscript, and to Dr. Alexander A. Bachmanov and Dr. Danielle R. Reed for our fruitful discussions. I also thank Dr. Ken-ichiro Muto and Dr. Kazuki Yoshioka, Department of Veterinary Anatomy, Faculty of Veterinary Medicine and Animal Sciences, Kitasato University, for their generous allowance for my stay in the U.S.A. This work is supported in part by NIH NIDCD Grand DC00882 (awarded to G. Beauchamp).

\section{REFERENCES}

1. Ariyasu, T., Matsumoto, S., Kyono, F., Hanaya, T., Arai, S., Ikeda, M. and Kurimoto, M. 2003. Taste receptor T1R3 is an essential molecule for the cellular recognition of the disaccharide trehalose. In Vitro Cell. Dev. Biol. Anim. 39: 80-88.

2. Bachmanov, A. A., Li, X, Reed, D. R., Ohmen, J. D., Li, S., 
Chen, Z., Tordoff, M. G., de Jong, P. J., Wu, C., West, D. B., Chatterjee, A., Ross, D. A. and Beauchamp, G. K. 2001. Positional cloning of the mouse saccharin preference (Sac) locus. Chem. Senses 26: 925-933.

3. Chandrashekar, J., Mueller, K. L., Hoon, M. A., Adler, E., Feng, L., Guo, W., Zuker, C. S. and Ryba, N. J. P. 2000. T2Rs function as bitter taste receptors. Cell 100: 703-711.

4. Demak, S., Rong, M., Yasumatsu, K., Kokrashvili, Z., Varadarajan, V., Zou, S., Jiang, P., Ninomiya, Y. and Margolskee, R. F. 2003. Detection of sweet and umami taste in the absence of taste receptor T1r3. Science 301: 850-853.

5. Dulac, C. 2000. The physiology of taste, vintage 2000. Cell 100: 607-610.

6. Hofer, D. and Drenckhahn, D. 1998. Identification of the taste cell G-protein, alpha-gustducin, in brush cells of the rat pancreatic duct system. Histochem. Cell Biol. 110: 303-309.6.

7. Kitagawa M., Kusakabe, Y., Miura, H., Ninomiya, Y. and Hino A. 2001. Molecular genetic identification of a candidate receptor gene for sweet taste. Biochem. Biophy. Res. Comm. 283: 236-242.

8. Li, X., Staszewski, L., Xu, H., Durick, K., Zoller, M. and Adler, E. 2002. Human receptors for sweet and umami taste. PNAS 99: 4693-4696.

9. Lindemann, B. 1996. Taste reception. Physiol. Rev. 76: 718766.

10. Lindemann, B. 2001. Receptors and transduction in taste. Nature (Lond.) 413: 219-225.

11. Malaisse, W. J. 1998. The riddle of L-glucose pentaacetate insulinotropic action (Review). Int. J. Mol. Med. 2: 383-388.

12. Max, M., Shanker, Y. G., Huang, L., Rong, M., Liu. Z., Campagne, F., Weinstein, H., Damak, S. and Margolskee. R. F. 2001. Tas1r3, encoding a new candidate taste receptor, is allelic to the sweet responsiveness locus Sac. Nature Genetics 28: $58-63$.

13. Montmayeur, J. -P., Liberles, S. D., Matsunami, H. and Buck, L. B. 2001. A candidate taste receptor gene near a sweet taste locus. Nature Neuroscience 4: 492-498.

14. Nelson, G., Chandrashekar, J., Hoon, M. A., Feng, L., Zhao, G., Ryba, N. J. P. and Zuker C. S. 2002. An amino-acid taste receptor. Nature (Lond.) 726: 199-202.

15. Nelson, G., Hoon, M. A., Chandrashekar, J., Zhang, Y., Ryba, N. J. P. and Zuker, C. S. 2001. Mammalian Sweet Taste receptors. Cell 106: 381-390.

16. Reed, D. R., Li, S., Li, X., Huang, L., Tordoff., M. G., StarlingRoney, R., Taniguchi, K., West, D. B., Ohmen, J. D., Beauchamp, G. K. and Bachmanov, A. A. 2004. Polymorphisms in the taste receptor gene (Tas1r3) region are associated with saccharin preference in 30 mouse strains. J. Neurosci. 24: 938 946.

17. Sainz, E., Korley, J. N., Battey, J. F. and Sullivan, S. L. 2001. Identification of a novel member of the T1R family of putative taste receptors. J. Neurochem. 77: 896-903.

18. Temmusi, P. A. 2002. Why are sweet proteins sweet? Interaction of Brazzein, monellin, and thaumatin with the T1R2-T1R3 receptor. FEBS Letters 526: 1-4. 